\title{
Características clínico-epidemiológicas del Síndrome de Guillain Barré en tres hospitales de Piura, 2018-2019.
}

Clinical and epidemiological characteristics of Guillain Barré Syndrome in three hospitals in Piura, Perú, 2018-2019.

\author{
Fiorela E. Solano ${ }^{1,2, a}$
}

\section{RESUMEN}

El síndrome de Guillain-Barré (SGB) es una polirradiculoneuropatía inflamatoria aguda con presentaciones clínicas diversas clasificadas como variantes clínicas, sobre las cuales se tiene escasa infomación en nuestra región. Objetivo: Determinar las características epidemiológicas y variantes clínicas del SGB. Materialy Métodos: Estudio descriptivo transversal retrospectivo. Se revisaron las historias clínicas de los casos sospechosos de SGB en tres hospitales de Piura, entre 2018-2019. Se incluyeron todos los casos sin límite de edad, excluyéndose únicamente aquellos con duda diagnóstica o historias incompletas. Los datos se analizaron mediante STATA vs 15.0. Resultados: De 123 casos, $61 \%$ fueron varones, con una edad promedio de 37 años. El 36,59\% de los casos no presentaban antecedentes clínicos. La comorbilidad más frecuente fue Hipertensión Arterial (HTA). Sólo $40 \%$ de los casos fueron confirmados. El 78\% se trató con inmunoglobulina. La variante más frecuente fue Poliradiculopatía Desmielinizante Inflamatoria Aguda (AIDP, sigla en inglés). Conclusiones: El SGB se presentó con mayor frecuencia en el sexo masculino y la forma clásica fue la variante más frecuente seguida de la regional. La mayoría de casos fueron clasificados como leves, con un grado promedio de discapacidad de 3 . Se recomiendan estudios sobre la asociación entre cada variante clínica y las características clínicas de la enfermedad.

PALABRAS CLAVE: Epidemiología, Síndrome De Guillain-Barré, Síndrome de Miller-Fisher

\section{SUMMARY}

Guillain-Barré syndrome (GBS) is an acute inflammatory polyradiculoneuropathy with diverse clinical presentations classified as clinical variants, about which little information is available in our region. Objective: To determine the epidemiologic characteristics and the clinic variants of Guillain-Barre Syndrome. Material and Methods: Retrospective cross-sectional study. The clinical records of the suspected cases of GBS in three hospitals in Piura (Northern Perú), between 2018 to 2019, were reviewed. Those with doubtful diagnosis or incomplete histories were excluded. Data were analysed through STATA vs 15.0. Results: Of 123 cases, $61 \%$ were male, with an average age of 37 , and $36.59 \%$ had no previous clinical manifestations. The most frequent comorbidity was high blood pressure.

Facultad de Medicina Humana, Universidad Privada Antenor Orrego. Trujillo, Perú.

Sociedad Científica de estudiantes de Medicina, Universidad Nacional de Piura. Piura, Perú.

Médico cirujano, Docente auxiliar. 
Only $40 \%$ of the cases were confirmed. $78 \%$ were treated with immunoglobulin. The most frequent variant was AIDP. Conclusions: Guillain-Barre Syndrome occurred more frequently in males and the classical form was the most frequent variant, followed by the regional form. Most cases presented a mild severity level, with 3 as the average degree of disability. Studies on the association between each clinical variant and the clinical characteristics of the disease are recommended.

KEYWORDS: Axonal, Epidemiology, Guillain-Barre Syndrome, variant.

\section{INTRODUCCIÓN}

El Síndrome de Guillain Barré (SGB) es una polirradiculoneuropatía inflamatoria aguda de progresión rápida que cursa con disautonomías y trastornos somáticos (1), afecta entre 1 y 2 de cada 100000 habitantes a nivel mundial, con preferencia del sexo masculino y con un aumento de incidencia del $20 \%$ cada 10 años $(2,3)$.

Tiene varias clasificaciones, entre la que se destaca por el nivel de afectación, donde se encuentran variantes como: Polirradiculoneuropatía desmielinizante inflamatoria aguda- AIDP; variante axonal que a su vez se subclasifica en Neuropatía axonal motora aguda- AMAN y Neuropatía axonal sensorio motora aguda- AMSAN; Pandisautonomía; Variantes regionales como Síndrome de Miller Fisher; Encefalitis de Bickerstaff brainstem; orofaríngea y Faríngeo cérvico braquial $(4,5)$. Cada una de ellas es distinta, probablemente por sus bases fisiopatológicas, como una mediación inflamatoria o un bloqueo de la conducción nerviosa e incluso lesión isquémica (6).

Su etiología no es clara, probablemente resulte del accionar del calcio dentro de la membrana axonal (7). Sin embargo, se conoce que se origina posterior a un proceso infeccioso, como enfermedad diarreica descrito en un estudio realizado en España o infección respiratoria previa tal como lo señaló un estudio realizado en Dinamarca. En estos dos estudios, se evidencia un porcentaje entre 27 a $30 \%$ sin infección precedente $(8,9)$. Otros estudios respaldan la hipótesis de su relación como un síndrome post vacunación, sin embargo, no son concluyentes. $(10,11)$.

El SGB puede ser letal, con complicaciones como ileo paralítico o insuficiencia respiratoria aguda debido a la retención de $\mathrm{CO} 2$, lo que desencadenaría en muerte $(12,13)$. A nivel mundial, la tasa de letalidad es variable, aunque en Perú se presenta una tasa del $3,5 \%(14)$
En Puerto Rico, el 72\% de los casos sospechosos fueron confirmados para SGB; lo que hace denotar el difícil diagnóstico de esta patología, considerando 3 días como mínimo para su confirmación, a través de Criterios de Brigthon o hallazgos en pruebas electromiográficas o de LCR (15).

En Perú, existió un brote epidemiológico en el año 2019 con un registro de aproximadamente 1100 casos, más del doble de los registrados el año anterior (16). Motivo por el cual se destinó un presupuesto para la actuación frente al SGB. A diferencia de años anteriores, Piura se encontró entre los departamentos con mayor prevalencia de la enfermedad $(14,17)$.

El aumento considerable de número de casos en Piura, así como su relación a procesos infecciosos, introduce la necesidad de estudios epidemiológicos en este departamento.

El objetivo de este estudio fue determinar las características clínico- epidemiológicas, y conocer los tipos de variantes clínicas del Síndrome de Guillain Barré en Piura.

\section{MATERIAL Y METODOS}

Se realizó un estudio descriptivo trasnversal a partir de datos registrados en historias clínicas de los años 2018 y 2019 de tres hospitales de Piura: Hospital de la Amistad Perú-Corea Santa Rosa II-2; Hospital de Apoyo Sullana II-2; Hospital Regional Cayetano Heredia III.

Se revisaron las historias clínicas de aquellos pacientes con sospecha de SGB notificados a epidemiología (código CIE 10 : G61.0), se incluyeron las historias clínicas de todos los pacientes sin excepción de edad, que presentaran evaluación neurológica por un especialista en neurología o neurointensivismo, que describían los criterios de Brighton; y aquellas sin evaluación neurológica con confirmación diagnóstica por laboratorio (disociación albúminocitológica) o por electromiografía (14). 
Se consideraron cuatro variantes de SGB: AIDP; Variante axonal (AMAN y AMSAN); Pandisautonomía; Variantes regionales (Síndrome de Miller Fisher; Encefalitis de Bickerstaff brainstem; orofaríngea y Faringeo cervico braquial); las cuales se identificaron según las características clínicas reportadas (5). Los pacientes con síntomas no compatibles con ninguna de las variantes anteriores, se incluyeron como una variante atípica.

Los datos fueron procesados y analizados en el programa STATA versión 15.0. Se usó distribución de frecuencias y porcentajes para las variables cualitativas y uso de media y rangos para la variable edad.

Este artículo fue generado por un registro secundario en la base de datos de los hospitales, se accedió a las historias clínicas solo para el reconocimiento de las variantes de Guillain Barré. En todo momento se consideró la confidencialidad de los datos. El documento fue revisado por el comité de ética institucional del Hospital de la Amistad Perú-Corea II-2 Santa Rosa.

\section{RESULTADOS}

De las 137 historias clínicas seleccionadas, 3 de ellas estaban duplicadas, 2 presentaban duda diagnóstica y 9 no cumplían con los criterios de selección propuestos. Se incluyeron 123 historias; donde predominaban los hombres en el $61,8 \%$ de los casos; provenientes de zona urbana $(70,4 \%)$ y con edad comprendida entre 30 a 59 años, con una media de 37,2 años. Asimismo, se encontró que la mayoría de la población eran desempleados o estudiantes (tabla $1)$.

Respecto a su procedencia, sólo 103 pacientes contaban con tal dato en la historia clínica, de los cuales, el $94,2 \%$ de los pacientes procedían del departamento de Piura; 3 casos de Tumbes, 1 caso de Lima, 1 de Trujillo y 1 de Lambayeque .Dentro de las provincias de Piura, procedían generalmente de Piura y Sullana con $42(40,8 \%)$ y $22(21,4 \%)$ casos respectivamente (gráfico 1).

Tabla 1. Características sociodemográficas de los pacientes con SGB.

\begin{tabular}{lcc}
\hline Característica sociodemográfica & n & \% \\
\hline Nacionalidad & 120 & 97,6 \\
Peruana & 3 & 2,4 \\
Extranjera & 37,19 & $(32,9-41,4)$ \\
Edad (años) & 23 & 18,7 \\
$0-11$ & 14 & 11,4 \\
12 a 17 & 16 & 13,0 \\
18 a 29 & 44 & 35,7 \\
30 a 59 & 26 & 21,2 \\
60 a más & & \\
Sexo & 47 & 38,2 \\
Femenino & 76 & 61,8 \\
Masculino & & \\
Empleo & 46 & 43,8 \\
Desempleo/Estudiante & 14 & 13,3 \\
Trabajo sedentario & 45 & 42,9 \\
Trabajo no sedentario & & \\
Zona de Procedencia & 34 & 29,6 \\
Rural & 81 & 70,4 \\
Urbana & & \\
Sede de recolección & 56 & 45,5 \\
Hospital de la Amistad Santa Rosa & 21 & 17,1 \\
Hospital de Apoyo -Sullana & 48 & 37.4 \\
Hospital Regional Cayetano Heredia & &
\end{tabular}




\section{Provincias de Piura}

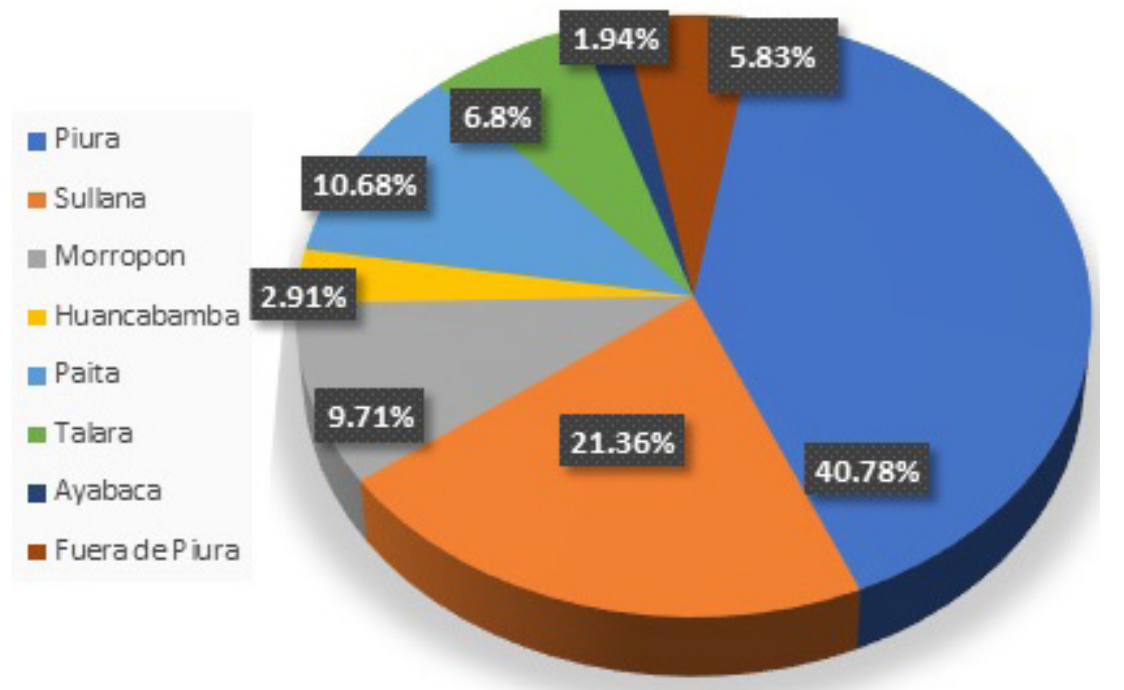

Gráfico 1. Provincias de procedencia de los pacientes con SGB

El $40 \%$ de los pacientes tenían un tiempo de enfermedad reciente, menor a 3 días, no se encontró antecedente clínico en el $36,6 \%$ de los casos. 3 pacientes presentaban por segunda vez la enfermedad. El $37,4 \%$ presentaban algún tipo de comorbilidad, dentro de las cuales, la hipertensión arterial seguida de gastropatía fueron las más frecuentes (tabla 2).

La mayoría de pacientes ingresaron por emergencia, sólo el 2,4\% ingresaron por consultorio externo. De los cuales, 58 pacientes ingresaron por referencia de algún establecimiento de salud $(47,2 \%)$ y sólo 11 pacientes $(8,9 \%)$ fueron contrareferidos.

De los 23 pacientes que contaban con otro tipo de antecedente clínico, se encontró mayor presencia de un síndrome febril inespecífico semanas previas a la aparición del síndrome (gráfico 2).

En cuanto al manejo de la enfermedad, sólo un caso no fue evaluado por especialista neurológico y sólo un caso fue evaluado por telemedicina. 107 pacientes recibieron tratamiento $(86,9 \%)$; se evidenciaron dos tipos de tratamiento: plasmaféresis $(21,1 \%)$ e inmunoglobina $(78,9 \%)$. El inicio promedio para el tratamiento fue de 14 días $(91,6 \%)$, una historia no se consideró por no indicar tiempo de enfermedad.
Cincuenta casos $(40,7 \%)$ fueron confirmados con electromiografía o punción lumbar, mientras dos casos se confirmaron por ambos métodos.

En cuanto a las variantes clínicas, se reconoce a la AIDP como la de mayor frecuencia con 49,6\% de los casos, seguida de la variante atípica y axonal motora con $18,7 \%$ y $11,4 \%$ respectivamente. En menor frecuencia las demás variantes (gráfico 4).

Los pacientes ingresaron con un grado de discapacidad 3-4 según la Escala de Hughes; asimismo la mayoría (80,5\%) contaba con una estancia hospitalaria menor a 15 días. Dentro de las complicaciones de la enfermedad, se encontró insuficiencia respiratoria en el 14,6\%, seguida por la hipertransaminemia en 17 casos $(13,8 \%)$ y trombosis en el 6,5\% de casos (gráfico 5).

El 18,3\% de los casos requirió oxígeno, donde 14 de ellos $(60,3 \%)$ requirieron de ventilación mecánica. El ingreso en una unidad crítica ocurrió en el 13 de los casos. $86,1 \%$ de pacientes fueron dados de alta, se evidenciaron 3 muertes por insuficiencia respiratoria, 2 de los cuales requerían ventilación mecánica y no se pudo concretar (tabla 3 ). 
Tabla 2. Características clínicas de los pacientes con SGB.

\begin{tabular}{|c|c|c|}
\hline Característica clínica & $\mathbf{n}$ & $\%$ \\
\hline \multicolumn{3}{|l|}{ Tiempo de enfermedad } \\
\hline$\leq 3$ días & 48 & 40,0 \\
\hline 4 a 7 días & 39 & 32,5 \\
\hline 8 a 14 días & 23 & 19,2 \\
\hline más de 14 días & 10 & 8,3 \\
\hline \multicolumn{3}{|l|}{ Antecedente clínico } \\
\hline No tiene & 45 & 36,6 \\
\hline Enfermedad diarreica & 27 & 22,0 \\
\hline Infección respiratoria alta & 25 & 20,3 \\
\hline Vacunación & 3 & 2,4 \\
\hline Otro & 23 & 18,7 \\
\hline \multicolumn{3}{|l|}{ Antecedente de SGB } \\
\hline No & 118 & 97,5 \\
\hline $\mathrm{Si}$ & 3 & 2,5 \\
\hline \multicolumn{3}{|l|}{ Tipo de comorbilidad } \\
\hline HTA & 22 & 29,7 \\
\hline Gastropatía & 9 & 12,2 \\
\hline DM2 & 7 & 9,5 \\
\hline Obesidad & 7 & 9,5 \\
\hline Dislipidemia & 5 & 6,7 \\
\hline Cardiopatía/arritmia & 5 & 6,7 \\
\hline Alergia & 5 & 6,7 \\
\hline Fibrosis pulmonar & 4 & 5,4 \\
\hline Artrosis/Artritis & 4 & 5,4 \\
\hline Hipotiroidismo/adenoma hipofisario & 2 & 2,7 \\
\hline Prostatismo/HBP & 2 & 2,7 \\
\hline Ansiedad & 1 & 1,4 \\
\hline ERC & 1 & 1,4 \\
\hline
\end{tabular}

\section{Otros antecedentes clínicos}

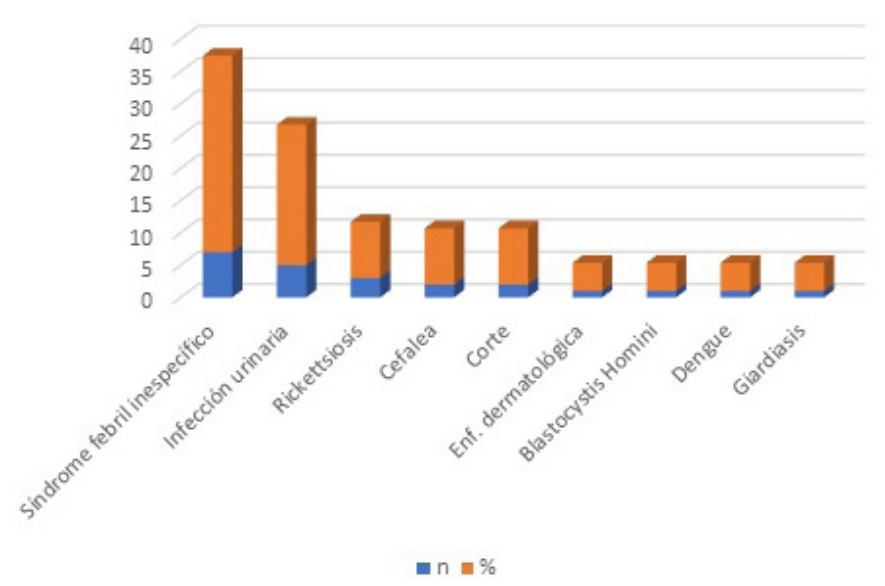

Gráfico 2. Distribución de otros antecedentes clínicos del SGB 


\section{Variantes clínicas}

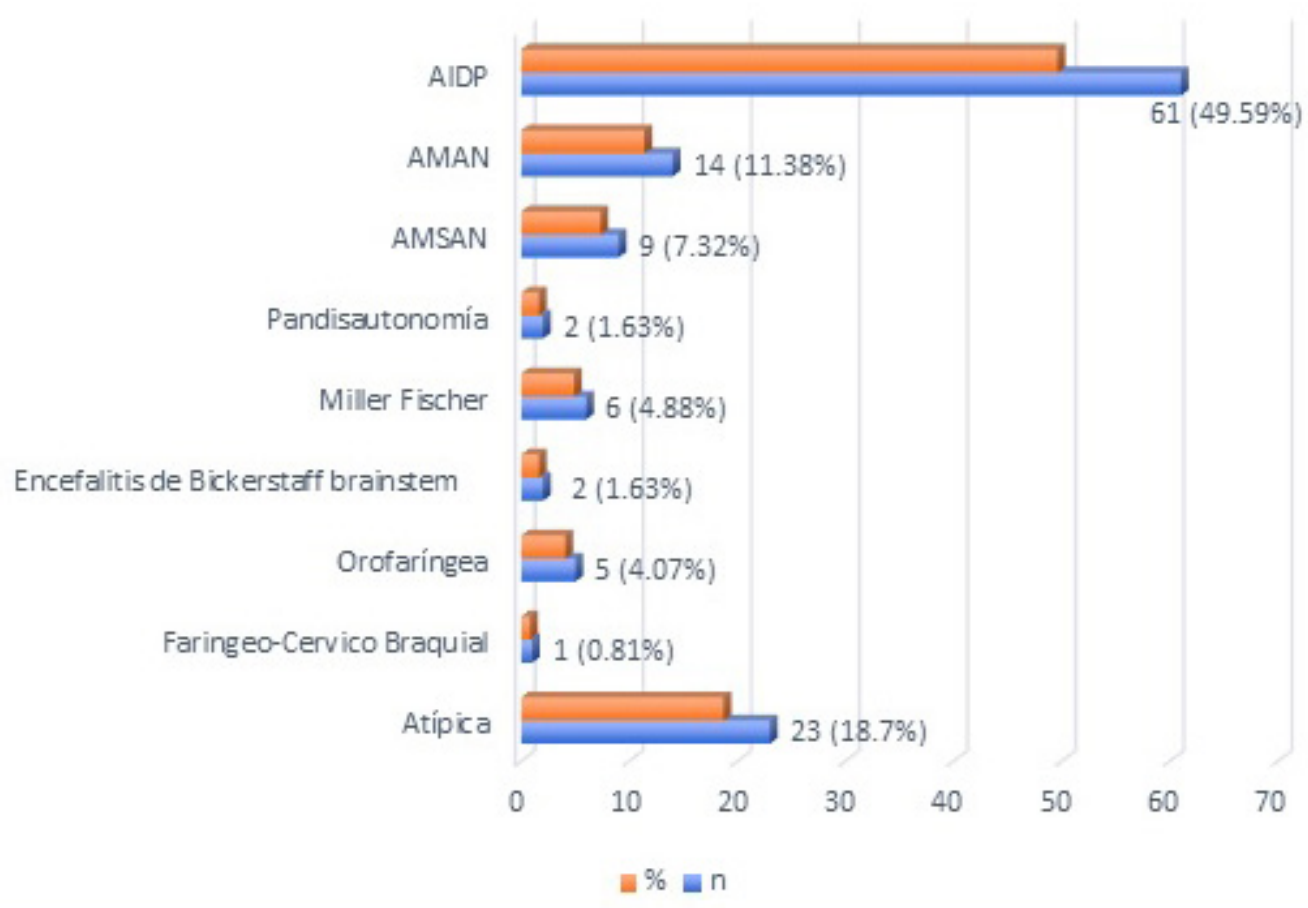

Gráfico 4. Tipo de variante clínica del SGB

\section{Tipos de complicaciones}

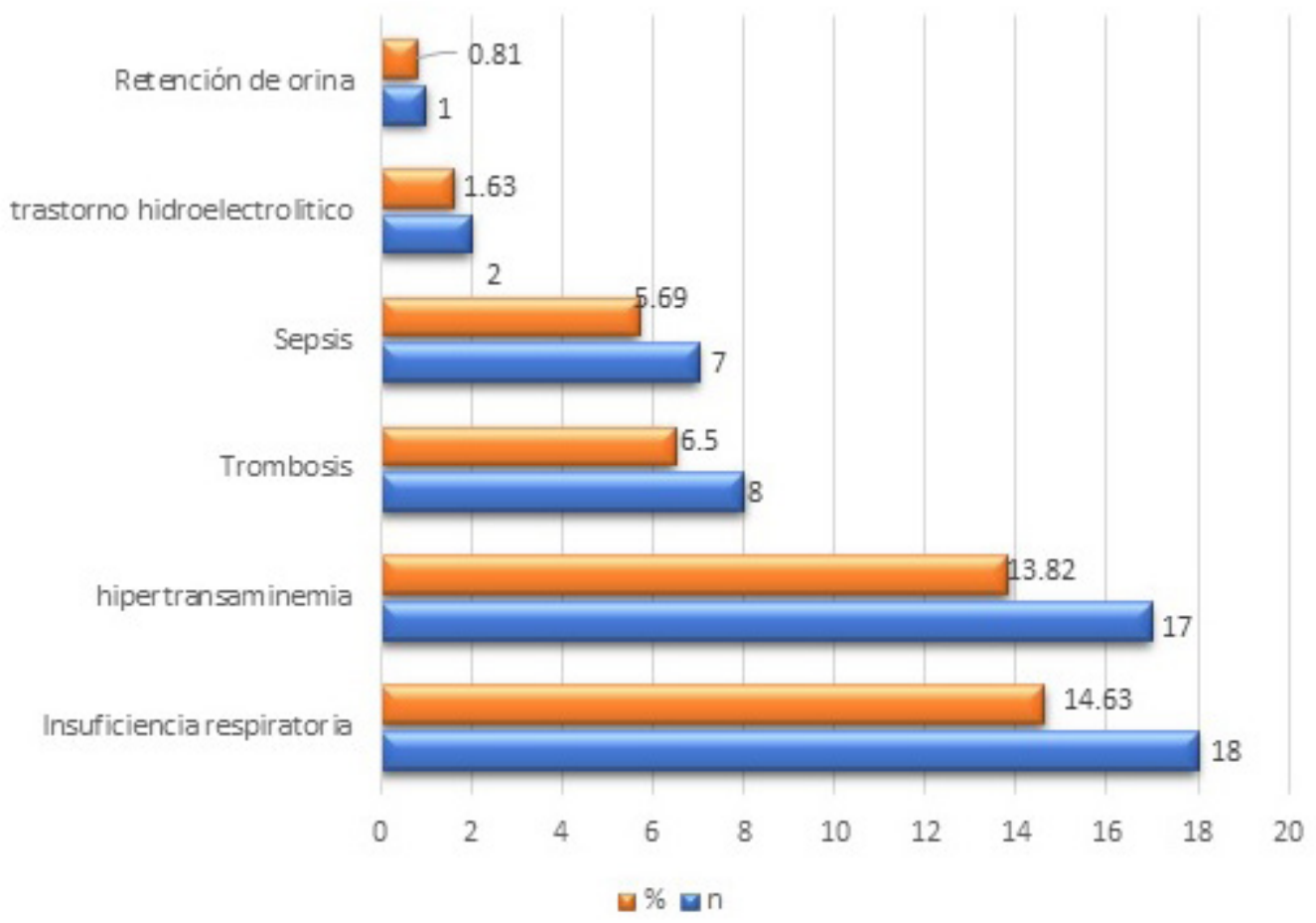

Gráfico 5. Tipos de complicaciones que presentan los pacientes con SGB. 
Tabla 3. Progresión del SGB

\begin{tabular}{|c|c|c|}
\hline & $\mathrm{n}$ & (\%) \\
\hline Tiempo de Hospitalización & \multicolumn{2}{|c|}{$14.33(0-151)$} \\
\hline 0-15 días & 99 & 80,5 \\
\hline 16 a más días & 24 & 19,5 \\
\hline \multicolumn{3}{|c|}{ Grado de discapacidad al ingreso } \\
\hline 0 & 0 & 0 \\
\hline 1 & 5 & 4,1 \\
\hline 2 & 27 & 22,0 \\
\hline 3 & 49 & 39,8 \\
\hline 4 & 34 & 27,6 \\
\hline 5 & 8 & 6,5 \\
\hline 6 & 0 & 0 \\
\hline \multicolumn{3}{|l|}{ Ingreso a unidad crítica } \\
\hline No & 107 & 87,0 \\
\hline $\mathrm{Si}$ & 16 & 13,0 \\
\hline \multicolumn{3}{|l|}{ Respirador artificial } \\
\hline No & 100 & 81,3 \\
\hline $\mathrm{Si}$ & 23 & 18,7 \\
\hline \multicolumn{3}{|l|}{ Ventilación mecánica } \\
\hline No & 109 & 88,6 \\
\hline $\mathrm{Si}$ & 14 & 11,4 \\
\hline \multicolumn{3}{|l|}{ Complicaciones } \\
\hline No & 91 & 74,0 \\
\hline $\mathrm{Si}$ & 32 & 26,0 \\
\hline \multicolumn{3}{|l|}{ Evolución clínica } \\
\hline Alta & 105 & 86,1 \\
\hline Referencia & 5 & 4,1 \\
\hline Retiro voluntario & 9 & 7,4 \\
\hline Muerte & 3 & 2,4 \\
\hline
\end{tabular}

11 pacientes no ingresaron a una unidad crítica a pesar de requerirlo, por presencia de negación de familiares en 4 casos y no disponibilidad de camas en casos.

\section{DISCUSIÓN}

En el presente estudio, se encontró una mayor frecuencia del SGB en el sexo masculino, ratificando lo que se ha demostrado anteriormente en otros estudios $(2,18,19)$ La edad promedio fue 37 años; en contraste con la presentación promedio de otros países de 50 años $(9,18,20)$; a nivel nacional, este Síndrome se presenta mayormente en jóvenes, tal como lo describe Munayco con estadísticas entre 2012 y 2017 (14), pero difiere de los hallazgos de los investigadores del Instituto Nacional de Ciencias Neurológicas (21).
La mayoría no reportaba un antecedente clínico, lo que concuerda con la literatura y contraria a lo reportado en el estudio de Sedano $(1,2,8,18)$. Asimismo, se encontraron antecedentes diferentes a un proceso infeccioso tales como cortes en piel y vacunación previa, esta última con asociación controversial según otros estudios $(10,11,22)$. La enfermedad diarreica tuvo una frecuencia ligeramente mayor sobre la infección respiratoria alta sin llegar a ser comparativo con estudios previos $(6,8)$.

Alrededor del $70 \%$ tuvieron un tiempo de enfermedad menor a 7 días, lo que concuerda con el estudio de Cheng (23). Las comorbilidades con mayor frecuencia encontradas fueron hipertensión arterial y gastropatía. En niños con SGB, se ha demostrado a la hipertensión como la disautonomía más frecuente con $44 \%$ de presencia; esto haría meritorio la realización de otros estudios que asocien estas comorbilidades con el síndrome (24).

Aunque los tres hospitales son centros de referencia, se obtuvo que el $47.15 \%$ de los pacientes ingresaron por referencia y solo el $8.94 \%$ fueron contrareferidos. Lo que demuestra la poca comunicación entre los establecimientos de salud y los centros hospitalarios en el margen de una enfermedad bajo vigilancia epidemiológica (25).

En cuanto al manejo de la enfermedad, solo un caso no fue evaluado por el especialista y solo un caso no recibió el tratamiento planteado por neurología, sin embargo, en ese caso, el paciente evolucionó favorablemente y fue dado de alta. El Instituto Nacional de Ciencias Neurológicas señala en su guía que el tratamiento debe priorizarse dentro de las primeras 2 semanas, debido a su mayor beneficio demostrado dentro de los 7 a 14 días de inicio del Síndrome $(26,27)$. En nuestro estudio, el tratamiento se inició mayormente dentro de los 14 días de inicio de los síntomas; con preferencia de la inmunoglobulina, la cual a pesar de no tener diferencias en cuanto a la eficacia con la plasmaféresis, se ha reconocido que no es eficaz en casos donde la plasmaféresis no hizo efecto con un $\mathrm{p}>0,15$ (28), además de ser una alternativa ante el no abastecimiento de la inmunoglobulina.

Más de la mitad de los casos contaban con el registro de una ficha epidemiológica. La confirmación diagnóstica ocurrió en el $40.65 \%$ de los casos; un porcentaje inferior al 70\% encontrado en Puerto Rico y podría ser mayor considerando que las tres sedes del estudio tienen acceso al recurso de la punción lumbar 
y más aun cuando uno de ellos puede realizar la prueba electromiográfica (15).

El grado de discapacidad al ingreso fue de 3, estudios anteriores nos señalan que entre 3 y 4 existe la mayor frecuencia en la escala de Hughes $(8,21)$ a diferencia del estudio de Arami que indica entre 2 a 3 (31). El tiempo de hospitalización promedio fue menor a 15 días, similar a lo que señaló Munayco y Guevara en sus respectivos estudios nacionales $(14,21)$.

El18,3\% de los casos requirieron oxígeno, yel 11,3\% ventilación mecánica, el cual es un porcentaje bajo con respecto a los estudios realizados en España $(6,8)$. Existió una frecuencia baja de los ingresos a unidades críticas y de complicaciones de la enfermedad, con un porcentaje de muerte de $2,46 \%$, lo que demuestra el buen pronóstico de la enfermedad y concuerda con los antecedentes $(14,32)$. La complicación de mayor frecuencia fue la insuficiencia respiratoria, la cual es común dentro de las enfermedades neurológicas con afectación neuromuscular (12).

En este estudio se reconocieron las variantes descritas en la clasificación que señala Wanleenuwat como las de mayor frecuencia, sin embargo, se demostró una alta presencia de variantes regionales y sobre todo de variantes atípicas con 33 casos en total. (5) Se demostró el primer lugar en frecuencia de la variante clásica seguida de la axonal y un porcentaje similar de la variante Miller Fisher con 4\% similar a otros estudios $(20,29,30,32)$. El estudio nacional de Guevara E señala a la variante axonal motora como la más frecuente a diferencia del presente estudio, cabe resaltar que el contexto de la realización de este estudio es un centro de referencia de casos neurológicos, sin embargo, también se reconoce a la variante Miller Fisher y la califica como una de las de menor frecuencia encontradas. (21) A pesar de que este estudio se realizó con los signos clínicos encontrados y descritos por el especialista en su nota clínica registrada en la historia, los resultados son congruentes con la literatura, en una revisión publicada por Leonhard SE se indica que cada variante es cada vez más "pura" o específica, por lo que sus signos y síntomas son característicos de cada variante (33).

Las limitaciones de estudio son: cuenta con sesgo de información ante la valoración de cada variante clínica, a pesar de tratar de tener objetividad en la clasificación, no se ha confirmado el tipo de variante, debido a que esta confirmación se realiza sólo electromiográficamente.
En conclusión, el presente estudio ofrece estadísticas importantes en el contexto del brote epidemiológico de Guillain Barré en Piura, demuestra una estrategia aceptable frente al SGB a nivel local y se demuestra la frecuencia de cada variante clínica donde predominó la variante clásica. Sin embargo, existieron deficiencias en el abordaje diagnóstico y en el sistema de referencias; asimismo la escasez de información a nivel nacional y local sobre las variantes clínicas llevan a que se recomienden estudios sobre la asociación entre cada variante clínica del síndrome y las características clínicas de la enfermedad; así como estudios prospectivos en la zona que permitan recolectar información sobre las secuelas a largo plazo.

\section{Correspondencia:}

Fiorela E. Solano,

A.H Santa Rosa Mz H6 Lote 11

Distrito 26 de octubre. Código postal: 20000, Piura, Perú.

Correo electrónico: elicenesolanozapata@outlook. com.

Declaración de financiamiento: El estudio fue financiado por la autora.

Conflicto de interés: No existe conflicto de interés en la investigación realizada.

Agradecimientos: Se agradece al Dr. Jorge Sigfredo Carmona Chávez por la colaboración en la elaboración del instrumento de trabajo y a la estudiante de medicina Alessia Rengifo por su colaboración en la redacción del manuscrito.

\section{REFERENCIAS BIBLIOGRÁFICAS.}

1. Organización Panamericana de la Salud. B o 1 e t í n Informativo de Guillain-Barre. Nicaragua: Organización Panamericana de la Salud; 2016.

2. Hao Y, Wang W, Jacobs BC, Qiao B, Chen M, Liu D, et al. Antecedent infections in Guillain-Barré syndrome: a single-center, prospective study. Ann Clin Transl Neurol. 2019; 6(12):2510-7.

3. Sejvar J, Baughman A, Wise M, Morgan OW. Population incidence of Guillain-Barre syndrome: a systematic review and meta-analysis. Neuroepidemiology. 2011; 36 (2):123-33.

4. Crovetto-Romero L. Variantes clínicasneurofisiológicas del síndrome de Guillian Barre. Rev Per Neurol. 1996;2(2-3):64-72.

5. Wanleenuwat $P$, Iwanowski $P$, Kozubski W. Antiganglioside antibodies in neurological diseases. J Neurol Sci. 2020; 408:116576.

6. Berciano J, Orizaola P, Gallardo E, Pelayo-Negro 
AL; Sánchez-Juan P; Infante $\mathrm{J}$ et al. Very early Guillain-Barre syndrome: A clinicalelectrophysiological and ultrasonographic study. Clin Neurophysiol Pract. 2020;5:1-9.

7. Kaida K.Guillain-Barré Syndrome. En: Sango K, Yamauchi J, Ogata T, Susuki K (editors). Myelin: Basic and Clinical Advances. Primera edición. Singapore: Springer Singapore; 2019. p. 323-31.

8. Sedano MJ, Orizaola P, Gallardo E, García A, PelayoNegro AL, Sánchez-Juan $P$ et al. A unicenter, prospective study of Guillain-Barre syndrome in Spain. Acta Neurol Scand. 2019;139(6):546-54

9. Al-Hakem H, Sindrup S, Andersen H, De la Cour CD, Lassen LL, Van den Berg B et al. GuillainBarre syndrome in Denmark: a population-based study on epidemiology, diagnosis, and clinical severity. J Neurol. 2019;266(2):440-9. 10. Chen $\mathrm{Y}$, Zhang J, Chu X,Xu Y, Ma F. Vaccines and the risk of Guillain-Barre syndrome. Eur J Epidemiol. 2020;35(4):363-370.

11. Wachira V, Peixoto H, De-Oliveira M. Systematic review of factors associated with the development of Guillain-Barre syndrome 2007-2017: what has changed? Trop Med Int Health. 2019;24(2):132-42.

12. Racca F, Vianello A, Mongini T, Ruggeri $P$, Versaci A, Vita GL et al. Practical approach to respiratory emergencies in neurological diseases. Neurol Sci. 2020;41(3):497-508. 13. Lee K, Ho T, Lee J, Lin LF, Chang WC, Shih CC et al. Paralytic ileus as the presenting symptom for Guillain-Barre syndrome: a case report. J Int Med Res. 2020; 48(4): 300060519893169.

14. Munayco C, Soto-Cabezas M, Reyes M, AricaGutierrez JA, Napanga-Saldaña O. Epidemiología del síndrome de Guillain-Barré en el Perú. Rev Perú Med Exp Salud Publica. 2019;36(1):10-16.

15. Major CG, Dirlikov E, Medina NA, Lugo-Robles R, Matos D, Muñoz-Jordán J et al. Implementation and Evaluation of Guillain-Barre Syndrome Surveillance in Puerto Rico during the 2016 Zika Virus Epidemic. P R Health Sci J. 2018; 37: S85-s92.

16. Organización Panamericana de la Salud. La OPS/ OMS colabora con el Ministerio de Salud ante el brote de Guillain-Barré. Perú: Organización Panamericana de la Salud; 2019.[Citado el 16 de enero del 2020]. Disponible en: https://www.paho.org/per/index. php?option $=$ com_content $\&$ view $=$ article $\& i d=$ 4324:la-ops-oms-colabora-con-el-ministerio-desalud-ante-el-brote-de-guillain-barre \&Itemid $=0$.

17. Ministerio de Salud. Plan de respuesta y rehabilitación del Ministerio de Salud frente al Síndrome de Guillain Barré para el año 2019. Lima: Ministerio de Salud; 2019.

18. Doets AY, Verboon C, Van-den-Berg B, Harbo T, Cornblath DR, Willison $\mathrm{HJ}$ et al. Regional variation of Guillain-Barre syndrome. Brain. 2018;141(10):
2866-77.

19. Dirección General de Epidemiologia. Situación de Guillain Barre, Perú al SE 02 - 2020. Lima: Dirección General de Epidemiología, Ministerio de Salud; 2020.

20. Granieri E, Andreasi N, De Martin P, Govoni V, Castellazzi M, Cesnik Eet al. Incidence study of Guillain-Barre syndrome in the province of Ferrara, Northern Italy, between 2003 and 2017. A 40-year follow-up. Neurol Sci. 2019;40(3):603-9.

21. Guevara-Silva E, Castro-Suárez S, Caparó-Zamalloa C, Cortez-Escalante J, Meza-Vega M. Características clínicas y respuesta al recambio plasmático terapéutico en los pacientes con síndrome de Guillain Barré. Rev Peru Med Exp Salud Pública. 2021;38(1):89-94.

22. Yoshikawa H. [Epidemiology of Guillain-Barré Syndrome]. Brain Nerve. 2015; 67(11):1305-11.

23. Cheng Q, Jiang G, Fredrikson S, Link H, De PedroCuesta J. Epidemiological surveillance of GuillainBarre syndrome in Sweden, 1996-1997. Network members of the Swedish GBS Epidemiology Study Group. Acta Neurol Scand. 2000;101(2):104-10

24. Watson L, Aziz M, Vassallo G, D Plant N, A Webb $\mathrm{NJ}$ et al. Bladder dysfunction and hypertension in children with Guillain-Barre syndrome. Pediatr Nephrol. 2014;29(9):1637-41.

25. Sansó F. La referencia y la contrarreferencia como expresión de la interrelación policlínico-hospital. Rev Cubana Med Gen Integr. 2002; 18(3): 236-238. [Citado el 8 de marzo del 2020] Disponible en: http:// scielo.sld.cu/scielo.php? script $=$ sci_arttext\&pid= S0864-21252002000300015\&lng=es.

26. Instituto Nacional de Ciencias Neurológicas. Guía de práctica clínica para el diagnóstico y tratamiento del paciente con síndrome del Guillian Barré. Guía en versión extensa. Lima: Instituto Nacional de Ciencias Neurológicas-Ministerio de Salud; 2018.

27. Pham H, Schwartz J. Therapeutic Plasma Exchange in Guillain-Barre Syndrome and chronic inflammatory demyelinating polyradiculoneuropathy. Presse Med. 2019;48(11):338-46.

28. Shalem D, Shemer A, Shovman O, Shoenfeld Y, Kivity S. The Efficacy of Intravenous Immunoglobulin in Guillain-Barré Syndrome: The Experience of a Tertiary Medical Center. Isr Med Assoc J. 2018;20 (12):754-60.

29. Jain R, Kookna J, Srivastva T, Jain R. Guillain-Barre Syndrome in Indian Population: A Retrospective Study. J Assoc Physicians India. 2019;67(4):56-9.

30. Uncini A, Ippoliti L, Shahrizaila N, Sekiguchi Y, Kuwabara S. Optimizing the electrodiagnostic accuracy in Guillain-Barre syndrome subtypes: Criteria sets and sparse linear discriminant analysis. Clin Neurophysiol. 2017;128(7):1176-83.

31. Arami M, Yazdchi M, Khandaghi R. Epidemiology, 


\section{Solano Fiorela E.}

and characteristics of Guillain-Barré syndrome in the northwest of Iran. Ann Saudi Med. 2006;26(1):22-7.

32. Baskar D, Amalnath D, Mandal J, Dhodapkar R, Vanathi K. Antibodies to Zika virus, Campylobacter jejuni and gangliosides in Guillain-Barre syndrome: A prospective single-center study from southern India. Neurol India. 2018;66(5):1324-31.
33. Leonhard SE, Mandarakas MR, Gondim FAA, Bateman K, Ferreira MLB, Cornblath DRet al. Diagnosis and management of Guillain-Barré syndrome in ten steps. Nat Rev Neurol. 2019; 15(11):671-683.

Recibido: 26/08/2020

Aceptado: 09/08/2021 